\title{
ESR investigation of hydrogen and deuterium atoms in impurity-helium solids
}

\author{
S.I. Kiselev, V.V. Khmelenko, E.P. Bernard, and D.M. Lee \\ Laboratory of Atomic and Solid State Physics \\ Cornell University, Ithaca, NY 14853-2501, USA \\ E-mail: epb22@cornell.edu
}

Received December 19, 2002

\begin{abstract}
Impurity-helium solids (Im-He solids) are porous solids created by injecting a beam of mixed helium and impurity gases into superfluid ${ }^{4} \mathrm{He}$. In this work we use electron spin resonance (ESR) techniques to investigate Im-He solids containing atoms and molecules of hydrogen and/or deuterium. We have performed studies of low temperature $(T \sim 1.35 \mathrm{~K})$ tunnelling chemical reactions in which deuterium atoms replace the hydrogen atoms bound in $\mathrm{H}_{2}$ or HD molecules to produce large (up to $7.5 \cdot 10^{17} \mathrm{~cm}^{-3}$ ) and relatively stable concentrations of free hydrogen atoms. The time dependence of $\mathrm{H}$ and $\mathrm{D}$ atom concentrations has been investigated for $\mathrm{Im}-\mathrm{He}$ samples with different initial ratios of hydrogen and deuterium ranging from 1:20 to 1:1.The satellite ESR lines associated with the dipolar coupling of electron spins of $\mathrm{H}$ and $\mathrm{D}$ atoms to the nuclear moments of the hydrogen nuclei found in neighboring molecules have been observed in Im-He solids. The forbidden hyperfine transition of atomic hydrogen involving the mutual spin flips of electrons and protons has also been observed.
\end{abstract}

PACS: 67.40.Yv, 67.40.Mj, 61.10.Eq, 61.46.+w

\section{Introduction}

Investigations of hydrogen and deuterium atoms, stabilized in solid matrices at low temperatures, have attracted the attention of scientists for many years. Possible quantum effects associated with these systems are of special interest. Quantum behavior is expected when the thermal de Broglie wavelength of the atoms becomes comparable with their interparticle spacing. For the case of $\mathrm{H}$ atoms in the gas phase, this condition is satisfied for a concentration of $2.6 \cdot 10^{18} \mathrm{~cm}^{-3}$ at a temperature of $30 \mathrm{mK}$ [1]. For the case of a solid phase, the temperature for the onset of any quantum effect may be lower because of the possibility of a larger effective mass. It is always desirable for investigations of quantum behavior to generate the highest possible concentrations of $\mathrm{H}$ or $\mathrm{D}$ atoms in solid matrixes. Unfortunately, from the earliest investigations of $\mathrm{H}$ atoms in solid $\mathrm{H}_{2}$, it became clear that some tunnelling processes and molecular recombination could lead to a decrease in the concentration of stabilized $\mathrm{H}$ atoms [2]. The detailed investigations of the processes of quantum diffusion and tunnelling reactions of $\mathrm{H}$ atoms in solid $\mathrm{H}_{2}$ at $T=1.35-4.2 \mathrm{~K}$ were performed by Ivliev et al. [3] and later by Miyazaki et al. [4-6]. They established that the decay of H atoms could take place in solid $\mathrm{H}_{2}$ by tunnelling migration, in which $\mathrm{H}$ atoms tunnel through a chain of $\mathrm{H}_{2}$ molecules according to the reaction

$$
\mathrm{H}+\mathrm{H}_{2} \rightarrow \mathrm{H}_{2}+\mathrm{H}
$$

thereby travelling through the solid $\mathrm{H}_{2}$ to recombine with another $\mathrm{H}$ atom. The reaction rate constant $k_{1}$, for reaction (1) was found to be $k_{1}=18 \mathrm{~cm}^{3} \cdot \mathrm{mol}^{-1} \cdot \mathrm{s}^{-1}$ at $T=4.2 \mathrm{~K}$ [4]. This value remains the same even when the temperature is lowered to $1.9 \mathrm{~K}$, confirming that tunnelling reactions are involved. The behavior of $\mathrm{D}$ atoms in solid $\mathrm{D}_{2}$ is different. The rate constant for the reaction

$$
\mathrm{D}+\mathrm{D}_{2} \rightarrow \mathrm{D}_{2}+\mathrm{D}
$$

is four orders of magnitude lower; $k_{2}=$ $=1.8 \cdot 10^{-3} \mathrm{~cm}^{3} \cdot \mathrm{mol}^{-1} \cdot \mathrm{s}^{-1}$ [7] leading to a much lower recombination rate. Therefore it is possible to create a larger concentration of $\mathrm{D}$ atoms in solid $\mathrm{D}_{2}$ as compared with concentrations of $\mathrm{H}$ atoms in solid $\mathrm{H}_{2}$ [7-9]. The exchange tunnelling reactions 


$$
\begin{aligned}
& \mathrm{D}+\mathrm{H}_{2} \rightarrow \mathrm{HD}+\mathrm{H} \\
& \mathrm{D}+\mathrm{HD} \rightarrow \mathrm{D}_{2}+\mathrm{H}
\end{aligned}
$$

lead to the possibility of creating high concentrations of $\mathrm{H}$ atoms in solid mixtures of $\mathrm{H}_{2}$ and $\mathrm{D}_{2}$ at low temperatures $[8,10,11]$. When an $\mathrm{H}$ atom is surrounded by shell of $\mathrm{D}_{2}$ molecules, it becomes very stable because it can neither migrate through the solid $\mathrm{D}_{2}$ nor react further with $\mathrm{D}_{2}$ by the reaction

$$
\mathrm{H}+\mathrm{D}_{2} \rightarrow \mathrm{HD}+\mathrm{D}
$$

at low temperatures, since it is an endothermic reaction. Gordon et al. [8,12] suggested that high concentrations of $\mathrm{H}$ atoms could be stabilized at low temperatures by means of reactions (3) and (4). In their approach, a gas mixture of hydrogen, deuterium and helium gas was transported through a radio frequency discharge onto the surface of superfluid He contained in a small beaker, at a temperature $1.5 \mathrm{~K}$. The jet of impurity and helium gases penetrates the surface of the superfluid He, and then forms a snow-like solid which settles to the bottom of the collection beaker. This solid became known as an Im-He solid [13,14]. In the case of heavy impurities, Im-He solids are built from a loosely connected aggregation of nanoclusters of impurities each surrounded by one or two layers of solid helium. These aggregates form extremely porous solids into which liquid helium easily and completely penetrates. This system, having the high thermal conductance and the high thermal capacitance of superfluid helium, allows preparation and storage of very high concentrations of stabilized atoms. To this date the structures of $\mathrm{Im}-\mathrm{He}$ solids for light impurities such as hydrogen and deuterium atoms and molecules are not fully determined. Our preliminary $\mathrm{x}$-ray investigations of $\mathrm{D}_{2}-\mathrm{He}$ samples showed the presence of nanoclusters of $\mathrm{D}_{2}$ in these solids [15]. Therefore it is reasonable to assume that the structure of Im-He solids formed from light impurities is similar to that of $\mathrm{Im}-\mathrm{He}$ solids formed from heavy impurities $\left(\mathrm{Ne}, \mathrm{N}_{2}, \mathrm{Kr}\right.$ ) [16,17]. In this work we have studied impurity-helium solids formed by light impurities, namely hydrogen and deuterium atoms and molecules. The method of electron spin resonance (ESR) was used for detailed studies of $\mathrm{H}$ and $\mathrm{D}$ atoms stabilized in Im-He solids. We have performed studies of the exchange tunnelling chemical reactions (3) and (4) to produce large (up to $7.5 \cdot 10^{17} \mathrm{~cm}^{-3}$ ) and relatively stable concentrations of $\mathrm{H}$ atoms. The kinetics of these reactions have been investigated for $\mathrm{Im}-\mathrm{He}$ samples formed by introducing gas mixtures with different initial ratios of $\mathrm{H}_{2}$ to $\mathrm{D}_{2}$, ranging from $1: 20$ to $1: 1$, into He II. We determined the exact positions of the $\mathrm{H}$ and $\mathrm{D}$ lines by using precise measurements of the magnetic field. Satellite ESR lines associated with the dipolar coupling of the electron spins of $\mathrm{H}$ and $\mathrm{D}$ atoms to the nuclear moments of hydrogen nuclei in neighboring molecules have been observed. This observation allows us to determine the distances between stabilized $\mathrm{H}$ or D atoms and neighboring $\mathrm{HD}$ or $\mathrm{H}_{2}$ molecules in $\mathrm{Im}-\mathrm{He}$ solids [18]. From the analysis of line widths and the saturation behavior of $\mathrm{H}$ and D signals, we estimate the spin-spin relaxation time $T_{2}$ and the spin-lattice relaxation time $T_{1}$ of $\mathrm{H}$ and $\mathrm{D}$ atoms in $\mathrm{Im}-\mathrm{He}$ solids.

\section{Experimental method}

The experiments were performed in a Janis cryostat with a variable temperature insert (VTI). The lower part of the cryostat was installed between the pole pieces of a Varian electromagnet for these ESR investigations. The home-made insert for the creation and investigation of $\mathrm{Im}-\mathrm{He}$ solids with stabilized atoms shown in Fig. 1 was placed into the VTI. The details of the experimental procedure were described in our previous work $[17,19]$. For sample preparation, a gas mixture of $\mathrm{H}_{2}, \mathrm{D}_{2}$, and $\mathrm{He}$ was transported from a room temperature gas handling system to the cryogenic region. To provide $\mathrm{H}$ and $\mathrm{D}$ atoms, high power radio frequency was applied to the electrodes around the quartz capillary carrying the mixed gases. The jet $\left(d N / d t \sim 5 \cdot 10^{19} \mathrm{~s}^{-1}\right)$ of impurity atoms and molecules as well as helium gas emerged from a small $(0.75 \mathrm{~mm})$ orifice and then penetrated the surface of superfluid helium in the collection beaker. The temperature during sample preparation was $1.5 \mathrm{~K}$. The liquid helium level in the beaker was maintained by a fountain pump connected to the main helium bath of the VTI. At the top of the beaker was a funnel that caught the sample as it emerged from the quartz capillary. A set of teflon blades was employed to scrape the sample from the funnel while the beaker was rotated so that the sample could fall to the bottom of the cylindrical part of the beaker. During a period of 10 minutes an impurity-helium solid sample with volume $0.35 \mathrm{~cm}^{3}$ was formed in the beaker. Following this process, the beaker with the sample was lowered into the ESR cavity, which was situated near the bottom of the VTI in the homogeneous field region of the electromagnet. We used a cylindrical cavity operating in a $T E_{011}$ mode. A ruby crystal was attached to the bottom of the cavity. The ruby was used as a secondary standard for the calibration of the measurements of the number of stabilized $\mathrm{H}$ and $\mathrm{D}$ atoms in $\mathrm{Im}-\mathrm{He}$ solids. The calibration of the absolute value of number of atoms was made by using a DPPH sample with a known number $\left(2.4 \cdot 10^{17}\right)$ of spins with measurements being carried out at $T=1.35 \mathrm{~K}$. Special measurements were made to determine the dependence of the signal of the small calibration sample of DPPH on the position along the axis of the cavity. This 


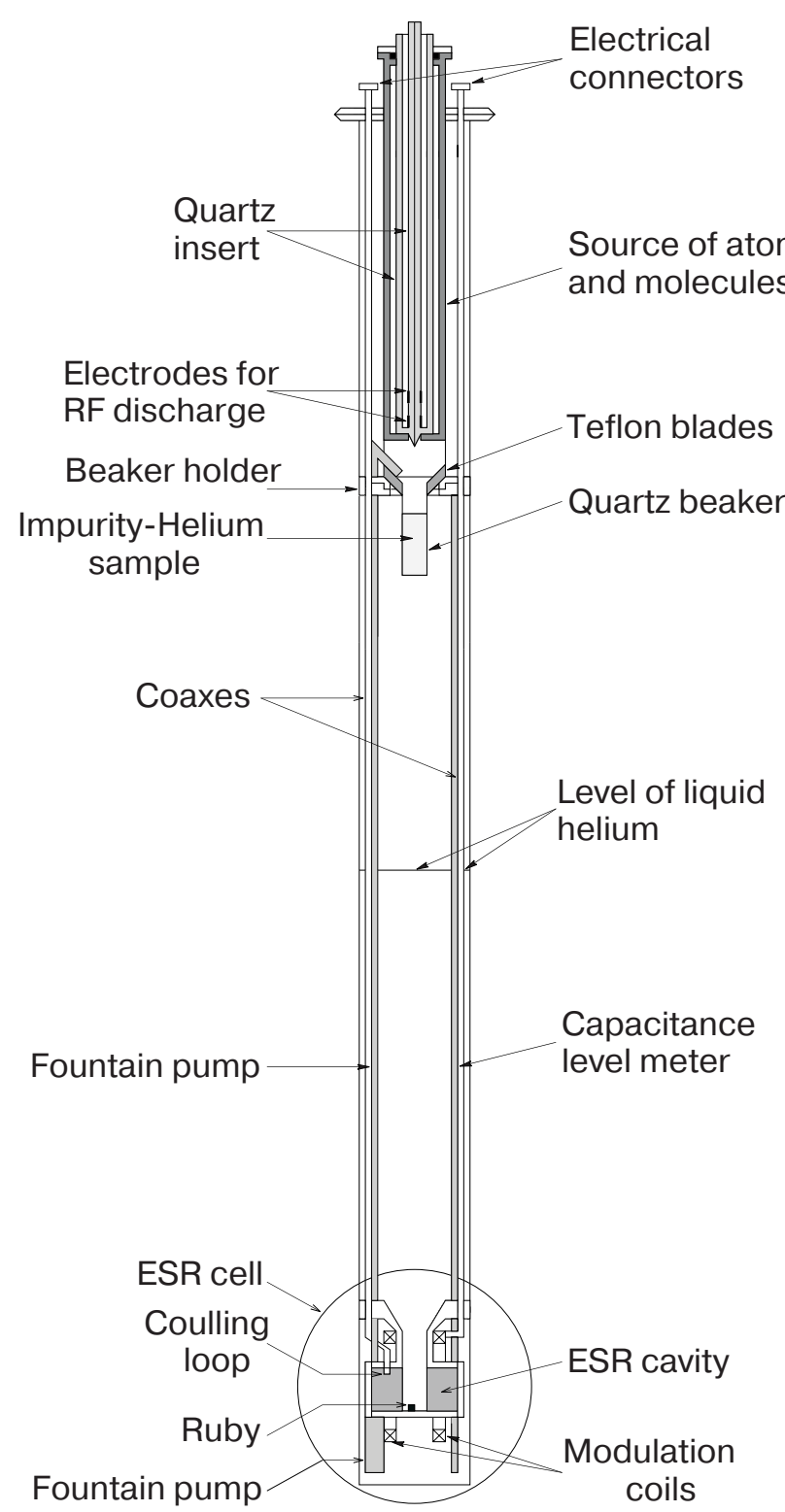

Fig. 1. Low temperature insert for Im-He sample preparation and ESR investigations. The quartz beaker is lowered into the ESR 9.12 GHz $T E_{011}$ resonant cavity for measurements after sample preparation.

dependence is consistent with the calculated distribution of the microwave magnetic field in the cavity. ESR signals were measured using a $\mathrm{CW}$ reflection homodyne spectrometer $\left(f_{r}=9.12 \mathrm{GHz}, f_{\text {mod }}=100 \mathrm{kHz}\right)$. All measurements have been done for $\mathrm{Im}-\mathrm{He}$ samples at temperatures of $1.35 \mathrm{~K}$ and $1.8 \mathrm{~K}$. A calibrated Ge thermometer was used for temperature measurements. Continuously pumping the VTI while supplying liquid helium from the main bath allows us to conduct long term investigations of the Im-He samples. In this paper we present reaction kinetics studies of $\mathrm{H}$ and $\mathrm{D}$ atoms which continued for $\sim 8$ hours at $T=1.35 \mathrm{~K}$.

\section{Results}

Figure 2 shows the ESR derivative spectra of $\mathrm{H}$ and $\mathrm{D}$ atoms in $\mathrm{Im}-\mathrm{He}$ solids prepared from an initial gas mixture in the ratio of $\mathrm{H}_{2}: \mathrm{D}_{2}: \mathrm{He}=1: 4: 100$. The positions of observed lines are shown in Table.

Table

Observed positions of ESR lines for $\mathrm{H}$ and $\mathrm{D}$ atoms in Im-He solids at $T=1.8 \mathrm{~K}$ and a frequency $9.12 \mathrm{GHz}$

\begin{tabular}{c|c|c}
\hline \hline Line & Transition $\left(\mathrm{F}, \mathrm{m}_{\mathrm{F}}\right)$ & Field, $\mathrm{G}$ \\
\hline \hline $\mathrm{H}_{1}$ & $(1,1) \leftrightarrow(0,0)$ & 2976.3 \\
$\mathrm{H}_{2}$ & $(1,0) \leftrightarrow(1,-1)$ & 3484.7 \\
$\mathrm{H}_{\mathrm{f}}$ & $(1,0) \leftrightarrow(0,0)$ & 3205.7 \\
$\mathrm{D}_{1}$ & $(3 / 2,3 / 2) \leftrightarrow(1 / 2,1 / 2)$ & 3171.7 \\
$\mathrm{D}_{2}$ & $(3 / 2,1 / 2) \leftrightarrow(1 / 2,-1 / 2)$ & 3247.4 \\
$\mathrm{D}_{3}$ & $(3 / 2,-1 / 2) \leftrightarrow(3 / 2,-3 / 2)$ & 3326.9 \\
\hline \hline
\end{tabular}

The allowed $\mathrm{H}$ and $\mathrm{D}$ lines are each accompanied by two satellite lines. The intensity of the small forbidden hydrogen line $\mathrm{H}_{f}$ is about 200 times smaller than that of the allowed hydrogen lines. Figure 3 shows the microwave power saturation behavior of $\mathrm{H}$ and $\mathrm{D}$ atoms in $\mathrm{Im}-\mathrm{He}$ solids. Unlike the behavior of $\mathrm{H}$ atoms produced by radiolysis in solid $\mathrm{H}_{2}$ [11], $\mathrm{H}$ atoms in $\mathrm{Im}-\mathrm{He}$ solids saturate at a larger microwave power $\sim 16 \mu \mathrm{W}$. ESR signals were normally measured at a microwave power $\sim 5 \mu \mathrm{W}$, significantly below the saturation limit.

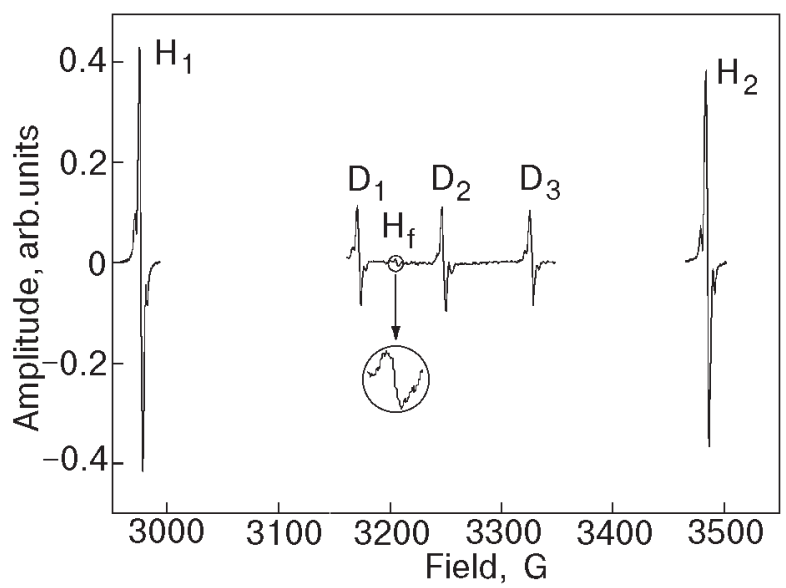

Fig. 2. ESR spectra of $\mathrm{H}$ and $\mathrm{D}$ atoms for an $\mathrm{Im}-\mathrm{He}$ solid prepared from the gaseous mixture $\mathrm{H}_{2}: \mathrm{D}_{2}: \mathrm{He}=1: 4: 100$. Spectra observed at $T=1.8 \mathrm{~K}, 182 \mathrm{~min}$ after sample collection. The width of each of the main hydrogen and deuterium lines is $3 \mathrm{G}$. Seven fold magnification of the forbidden line is shown in the inset. 


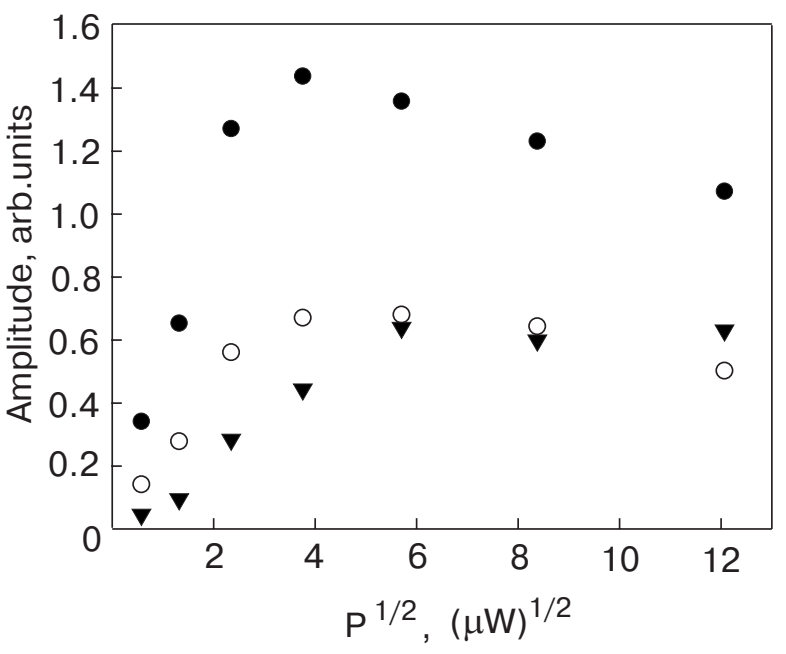

Fig. 3. Microwave power saturation behavior for different ESR lines of $\mathrm{H}$ and $\mathrm{D}$ atoms in $\mathrm{Im}-\mathrm{He}$ solid at $T=1.35 \mathrm{~K}$ (see Fig. 2): $\mathrm{H}_{1}(\bullet), \mathrm{D}_{1}(\mathrm{O}), \mathrm{H}_{f}(\boldsymbol{\nabla})$. Im-He solid was formed by initial gas mixture $\mathrm{H}_{2}: \mathrm{D}_{2}: \mathrm{He}=1: 4: 100$.

The spin-spin relaxation time $T_{2}$ is calculated from the line-width below saturation by means of the expression [20]:

$$
T_{2}=\frac{2}{3^{1 / 2} \gamma \Delta H_{p p}}
$$

where $\gamma$ is the gyromagnetic ratio, and $\Delta H_{p p}$ is the line width obtained from the peak to peak separation for the derivative of the ESR signal. The line width of ESR lines for $\mathrm{H}$ and $\mathrm{D}$ atoms was found to be $3 \mathrm{G}$ (see Fig. 2), leading to a value of $T_{2}=2.2 \cdot 10^{-8} \mathrm{~s}$ for $\mathrm{H}$ and $\mathrm{D}$ atoms in our $\mathrm{Im}-\mathrm{He}$ solids. The observed line width is far larger than the inhomogeneous broadening expected from our magnet $(\sim 0.1 \mathrm{G})$. We used a saturation method for determination of the spin-lattice relaxation time $T_{1}$ for $\mathrm{H}$ and $\mathrm{D}$ atoms in Im-He solids. For estimating $T_{1}$, we determined the dependence of the signal amplitude on the square root of microwave power as plotted in Fig. 3 for hydrogen and deuterium atoms. Values of $T_{1}$ were determined by means of an expression for the maximum values of the peak amplitude of the derivative signal [20]:

$$
T_{1}=\frac{1.98 \cdot 10^{-7} \Delta H_{p p}}{g H_{1}^{2}}
$$

where $g$ is the spectroscopic splitting factor. For $\mathrm{H}$ and D atoms $g \simeq 2$. We performed calculations of the microwave magnetic field according to the equation [20]:

$$
H_{1}^{2}=2 \cdot 10^{-3} P Q \eta \frac{V_{c}}{V s}
$$

where $P$ is the microwave power; $Q$ is the quality factor; $\eta$ is the filling factor; $V_{c}$ is the cavity volume, and
$V_{s}$ is the sample volume. When we substitute the geometric parameters for our cavity, expression (8) becomes $H_{1}^{2}=8 P$. From the plot in Fig. 3 we obtained the value of the power $P_{\max }=16 \mu \mathrm{W}$ for which the amplitude of the derivative of the ESR signal of the $\mathrm{H}$ atoms has a maximum value. From the expression (7) we then find a value of $T_{1}=(2.3 \pm 0.5) \cdot 10^{-3} \mathrm{~s}$ for $\mathrm{H}$ atoms in Im-He solids. For D atoms $\left(P_{\max }=25 \mu \mathrm{W}\right.$, see also Fig. 3) we find a value $T_{1}=(1.5 \pm 0.5) \cdot 10^{-3} \mathrm{~s}$. The relatively small values of $T_{1}$ for $\mathrm{D}$ and $\mathrm{H}$ atoms in Im-He solids show that the atoms are stabilized in solid clusters of mixtures $\mathrm{HD}$ and $\mathrm{D}_{2}$ molecules, rather than being isolated in liquid and solid helium. In latter case, the $T_{1}$ values should be much larger [21].

We also investigated the evolution of Im-He solids containing $\mathrm{H}$ and $\mathrm{D}$ atoms as well as $\mathrm{H}_{2}, \mathrm{HD}$, and $\mathrm{D}_{2}$ molecules in an attempt to maximize the $\mathrm{H}$ atom concentration. The investigations were performed for a variety of initial gas mixtures of $\mathrm{H}_{2}, \mathrm{D}_{2}$, and $\mathrm{He}$. The initial $\mathrm{H}_{2}: \mathrm{D}_{2}$ ratio was varied from $1: 20$ to $1: 1$. The ratio of the concentration of the impurity gases to the He gas in the mixtures was always equal to 1:20. The yields of $\mathrm{H}$ and $\mathrm{D}$ atoms leaving the radio frequency discharge were proportional to the concentrations of $\mathrm{H}_{2}$ and $\mathrm{D}_{2}$ in the initial gas mixture [12]. Figure 4 shows the time evolution of the $\mathrm{H}$ and $\mathrm{D}$ concentrations in Im-He solids formed by two different initial mixtures. Immediately after preparation of all the Im-He samples, a large enhancement of the concentration of $\mathrm{H}$ atoms relative to $\mathrm{D}$ atoms was observed compared with the ratio of $\mathrm{H}_{2}$ to $\mathrm{D}_{2}$ in the initial gas mixture. This fact indicates that at the earliest stages of sample preparation at $T=1.5 \mathrm{~K}$, a fast exchange tunnelling reaction leads to a large reduction in the number of $\mathrm{D}$ atoms and a corresponding increase in the number of $\mathrm{H}$ atoms. According to the calculation by Takayanagi et al. [22] the rate constant for reaction (3) is found to be $5.4 \cdot 10^{-2} \mathrm{~cm}^{3} \cdot \mathrm{mol}^{-1} \cdot \mathrm{s}^{-1}$, so that the enhancements of the $\mathrm{H}$ atom concentrations are attributed to this reaction with a time constant on the order of a few minutes for our samples. Later on, a steady increase of the ratio $\mathrm{H}: \mathrm{D}$ takes place over a period of hours, during the storage of our $\mathrm{Im}-\mathrm{He}$ sample at $T=1.35 \mathrm{~K}$. The kinetics of the changing concentrations of $\mathrm{H}$ and $\mathrm{D}$ atoms for the sample prepared from the initial gas mixture $\mathrm{H}_{2}: \mathrm{D}_{2}: \mathrm{He}=1: 20: 420$ is similar to that observed by Lukashevich et al. [10] and Miyazaki et al. [11] for atoms trapped in solid $\mathrm{H}_{2}-\mathrm{D}_{2}$ mixtures. The concentration of $\mathrm{H}$ atoms grows, but the concentration of $\mathrm{D}$ atoms is decaying, just as in our Im-He studies. At this stage we believe that the behavior of atoms in our solid is governed by reaction (4) with rate constant $1.9 \cdot 10^{-3} \mathrm{~cm}^{3} \cdot \mathrm{mol}^{-1} \cdot \mathrm{s}^{-1}$ [22] which further reduces the number of $\mathrm{D}$ atoms and in- 

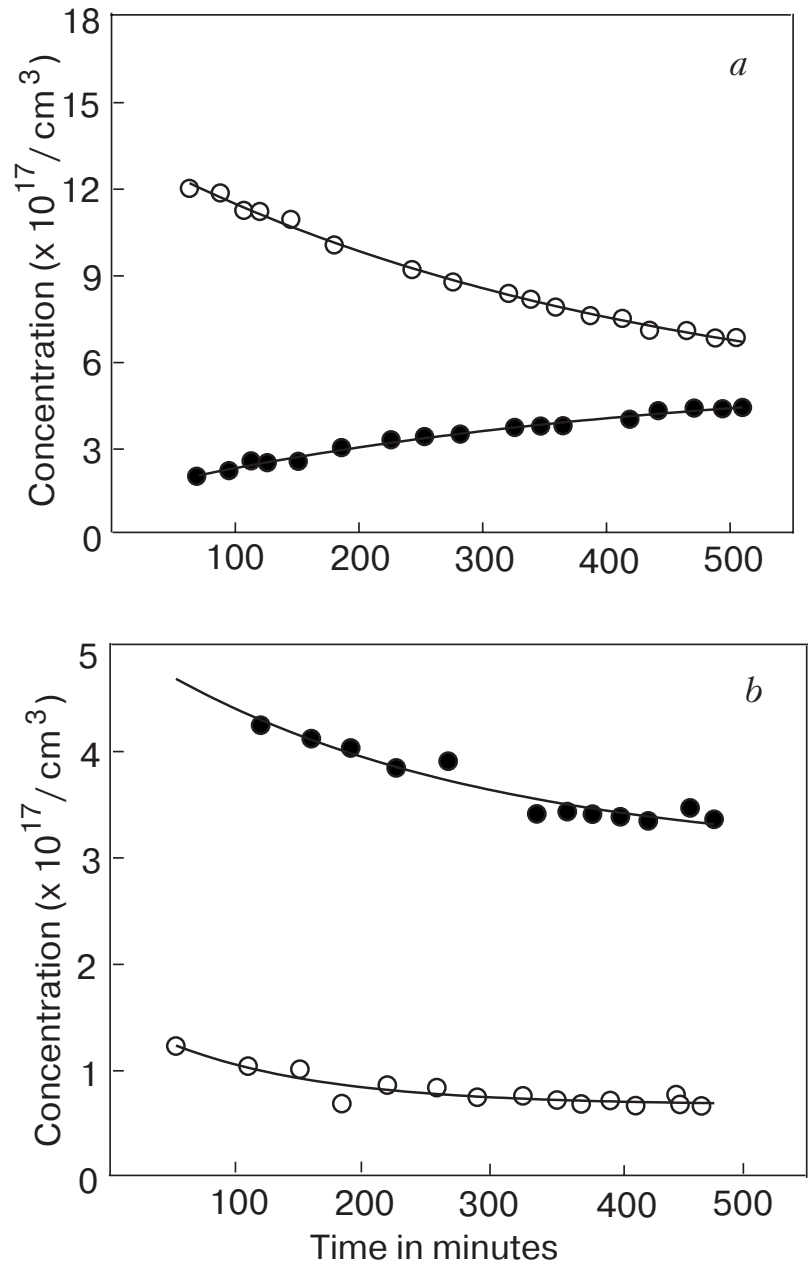

Fig. 4. Time dependence of the concentration of $\mathrm{H}$ atoms ( ) and D atoms (O) in Im-He solids at temperature $T=1.35 \mathrm{~K}$ prepared from different initial mixtures: $\mathrm{H}_{2}: \mathrm{D}_{2}: \mathrm{He}=1: 20: 420(a) ; \mathrm{H}_{2}: \mathrm{D}_{2}: \mathrm{He}=1: 2: 60(b)$.

creases the number of $\mathrm{H}$ atoms. Figure $4, a$ shows that the increase in the number of $\mathrm{H}$ atoms is smaller than the decrease in the number of $\mathrm{D}$ atoms. This observation could be explained by recombination of $\mathrm{H}$ atoms. In this process, the hydrogen atoms migrate through the solid via reaction (1) or the reaction

$$
\mathrm{H}+\mathrm{HD} \rightarrow \mathrm{HD}+\mathrm{H} \text {. }
$$

A succession of these reactions allows the transport of $\mathrm{H}$ atoms to neighboring positions in the $\mathrm{Im}-\mathrm{He}$ solid where they recombine to form $\mathrm{H}_{2}$ or $\mathrm{HD}$ molecules via the reactions:

$$
\begin{aligned}
& \mathrm{H}+\mathrm{D} \rightarrow \mathrm{HD} \\
& \mathrm{H}+\mathrm{H} \rightarrow \mathrm{H}_{2} .
\end{aligned}
$$

This mechanism is supported by the results of investigations of Im-He samples prepared from the gas mixture $\mathrm{H}_{2}: \mathrm{D}_{2}: \mathrm{He}=1: 2: 60$ (see Fig. $4, b$ ). In this sample the concentration of $\mathrm{H}_{2}$ and $\mathrm{HD}$ molecules is about one order of magnitude larger compared with the former sample, so therefore reactions (1) and (9) should accelerate the recombination processes (10) and (11). It can be seen that the decay of $\mathrm{D}$ atoms is more rapid and the $\mathrm{H}$ atom population also decays steadily throughout the experiment. The exchange tunnelling reactions can be used to produce very large concentrations of atomic hydrogen in our samples. As discussed earlier, these large concentrations could allow us to enter the regime where the thermal de Broglie wavelength becomes comparable to the spacing between $\mathrm{H}$ atoms, provided that the temperature is low enough. In our experiments we have investigated samples obtained from a variety of initial gas mixtures to determine the one which yields the highest hydrogen atom concentration. The experimental plots shown in Fig. 5 correspond to the $\mathrm{H}$ and $\mathrm{D}$ atom average concentrations after a storage time $\sim 500$ minutes following initial sample preparation. For the optimal gas mixture $\mathrm{H}_{2}: \mathrm{D}_{2}: \mathrm{He}=1: 4: 100$, the largest $\mathrm{H}$ atom concentration $(7.5 \pm 3.0) \cdot 10^{17} \mathrm{~cm}^{-3}$ was obtained. We are planning investigations with even longer storage times of this optimal sample to obtain the largest possible concentration of $\mathrm{H}$ atoms due to exchange tunnelling reactions.

\section{Conclusion}

The observation of satellite lines and the relatively short longitudinal relaxation times $T_{1}$ for $\mathrm{H}$ and $\mathrm{D}$ atoms in impurity-helium solids show that the $\mathrm{H}$ and $\mathrm{D}$ atoms are stabilized in clusters of solid mixtures of $\mathrm{H}_{2}, \mathrm{D}_{2}$, and HD. We did not observe any signatures of

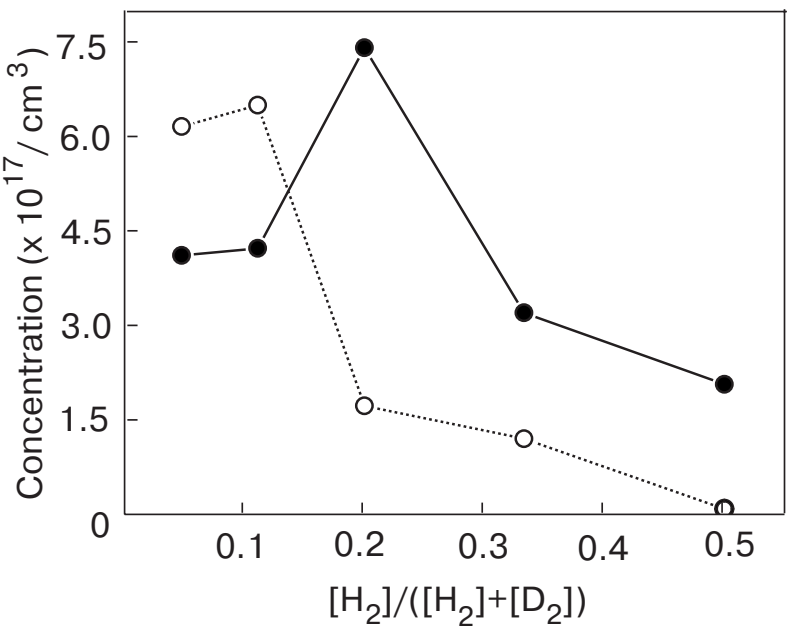

Fig. 5. The dependence of the average concentrations of $\mathrm{H}$ atoms (O) and D atoms (O) in Im-He solids on the fraction of hydrogen gas in the make up gas mixture, $\left[\mathrm{H}_{2}\right] /\left(\left[\mathrm{H}_{2}\right]+\left[\mathrm{D}_{2}\right]\right)$. For each point the concentrations were determined after a waiting period of 500 minutes following sample collection. 
single atoms perfectly isolated by helium. Observation of these isolated atoms requires the application of much lower microwave power levels to avoid saturation. Our spectrometer was not capable of providing such low powers.

The time dependence of the populations of $\mathrm{H}$ and $\mathrm{D}$ atoms in the $\mathrm{Im}-\mathrm{He}$ solids at $T=1.35 \mathrm{~K}$ proves the occurrence of the exchange tunnelling reaction $\mathrm{D}+\mathrm{HD}$ $\rightarrow \mathrm{H}+\mathrm{D}_{2}$. The exchange tunnelling reactions are capable of producing very high concentrations of hydrogen atoms. Our estimations from ESR line intensity measurements indicate that the largest concentration we have obtained is $(7.5 \pm 3.0) \cdot 10^{17} \mathrm{~cm}^{-3}$. For this concentration the onset of quantum overlap phenomena may be found at $T \sim 13 \mathrm{mK}$ which can be reached by means of a dilution refrigerator.

\section{Acknowledgements}

The authors are grateful to NASA for supporting this research via grant NAG 8-1445. We also thank P. Borbat, G. Codner, H. Padamsee, V. Shemelin, and R. Silsbee for extremely useful conversations and help with the experiments.

1. T.J. Greytak and D. Kleppner, Spin Polarized Hydrogen, New Trends in Atomic Physics, in: Les Houches Summer School - session XXXVIII (1982), G. Grinberg and R. Stone (eds.) Elsevier Science Publisher, B.V. (1984), p. 1127.

2. A.M. Bass and H.P. Broida, Formation and Trapping of Free Radicals, Academic Press, New York (1960).

3. A.V. Ivliev, A.Ya. Katunin, I.I. Lukashevich et al., JETP Lett. 36, 472 (1982).

4. T. Miyazaki, N. Iwata, K.-P. Lee, and K. Fueki, J. Phys. Chem. 93, 3352 (1989).

5. T. Kumada, S. Mori, T. Nagasaka, J. Kumagai, and T. Miyazaki, J. Low Temp. Phys. 122, 265 (2001).

6. T. Kumada, M. Sakakibara, T. Nagasaka, H. Fukuta, J. Kumagi, and T. Miyazaki, J. Chem. Phys. 116, 1109 (2002).

7. K.L. Lee, T. Miyazaki, K. Fueki, and K. Gotoh, J. Phys. Chem. 91, 180 (1987).
8. E.B. Gordon, A.A. Pelmenev, O.F. Pugachev, and V.V. Khmelenko, JETP Lett. 37, 282 (1983).

9. A.S. Iskovskih, A.Ya. Katunin, I.I. Lukashevich, V.V. Sklyarevskii, V.V. Suraev, V.V. Filippov, N.I. Filippov, and V.A. Shevtsov, Sov. JETP 64, 1085 (1986).

10. A.V. Ivliev, A.S. Iskovskih, A.Ya. Katunin, I.I. Lukashevich, V.V. Sklyarevskii, V.V. Suraev, V.V. Filippov, N.I. Filippov, and V.A. Shevtsov, JETP Lett. 38, 379 (1983).

11. H. Tsuruta, T. Miyazaki, K. Fueki, and N. Azuma, J. Phys. Chem. 87, 5422 (1983).

12. E.B. Gordon, A.A. Pelmenev, O.F. Pugachev, and V.V. Khmelenko, Fiz. Nizk. Temp. 11, 563 (1985) [Sov. J. Low Temp. Phys. 11, 307(1985)].

13. E.B. Gordon, V.V. Khmelenko, A.A. Pelmenev, E.A. Popov, and O.F. Pugachev, Chem. Phys. Lett. 155, 301 (1989).

14. E.B. Gordon, V.V. Khmelenko, A.A. Pelmenev, E.A Popov, O.F. Pugachev, and A.F. Shestakov, Chem. Phys. 170, 411 (1993).

15. S.I. Kiselev, V.V. Khmelenko, D.M. Lee, V. Kiryukhin, R.E. Boltnev, E.B. Gordon, and B. Keimer, J. Low Temp. Phys. 126, 235 (2002).

16. V. Kiryukhin, B. Keimer, R.E. Boltnev, V.V. Khmelenko, and E.B. Gordon, Phys. Rev. Lett. 79, 1774 (1997)

17. S.I. Kiselev, V.V. Khmelenko, D.M. Lee, V. Kiryukhin, R.E. Boltnev, E.B. Gordon, and B. Keimer, Phys. Rev. B65, 024517 (2002).

18. S.I. Kiselev, V.V. Khmelenko, and D.M. Lee, Phys. Rev. Lett. 89, 175301 (2002).

19. S.I. Kiselev, V.V. Khmelenko, D.M. Lee, and C.Y. Lee, J. Low Temp. Phys. 128, 37 (2002).

20. C.P. Poole, Electron Spin Resonance. A Comprehensive Treatise on Experimental Techniques, Interscience Publishers, John Wiley and Sons, New York, London, Sydney (1967).

21. M. Arndt, S.I. Kanorsky, A. Weis, and T.W. Hansch, Phys. Rev. Lett. 78, 1359 (1995).

22. T. Takayanagi, K. Nakamura, and S. Sato, J. Chem. Phys. 90, 1641 (1989). 